OPEN ACCESS

Edited by:

Eugenia Carrillo,

Instituto de Salud Carlos III, Spain

Reviewed by:

Sima Rafati,

Pasteur Institute of Iran, Iran

Ricardo Silvestre,

Instituto de Pesquisa em Ciências da

Vida e da Saúde (ICVS), Portugal

*Correspondence:

Claudia I. Brodskyn

brodskyn@bahia.fiocruz.br

Received: 27 April 2018 Accepted: 25 June 2018 Published: 26 July 2018

Citation:

Brodskyn Cl and Kamhawi S (2018) Biomarkers for Zoonotic Visceral Leishmaniasis in Latin America. Front. Cell. Infect. Microbiol. 8:245 doi: 10.3389/fcimb.2018.00245

\section{Biomarkers for Zoonotic Visceral Leishmaniasis in Latin America}

\author{
Claudia I. Brodskyn ${ }^{1 *}$ and Shaden Kamhawi ${ }^{2}$ \\ ${ }^{1}$ Instituto Gonçalo Moniz, Fiocruz Bahia, Salvador, Brazil, ${ }^{2}$ National Institute of Allergy and Infectious Diseases (NIAID), \\ National Institutes of Health, Bethesda, MD, United States
}

In Latin America, zoonotic visceral leishmaniasis (ZVL) arising from infection by L. infantum is primarily transmitted by Lutzomyia longipalpis sand flies. Dogs, which are chronic reservoirs of $L$. infantum, are considered a significant risk factor for acquisition of ZVL due to their close proximity to humans. In addition, as a vector-borne disease the intensity of exposure to vector sand flies can also enhance the risk of developing ZVL. Traditionally, IFN- $\gamma$ and IL-10 are considered as the two main cytokines which determine the outcome of visceral leishmaniasis. However, more recently, the literature has demonstrated that different mediators, such as lipid mediators (PGE-2, PGF-2 alfa, LTB-4, resolvins) and other important inflammatory and anti-inflammatory cytokines are also involved in the pathogenicity of ZVL. Analysis of a greater number of mediators allows for a more complete view of disease immunopathogenesis. Additionally, our knowledge has expanded to encompass different biomarkers associated to disease severity and healing after specific treatments. These parameters can also be used to better define new potential targets for vaccines and chemotherapy for ZVL. Here, we will provide an overview of ZVL biomarkers identified for both humans and dogs and discuss their merits and shortcomings. We will also discuss biomarkers of vector exposure as an additional tool in our arsenal to combat ZVL.

Keywords: zoonotic visceral leishmaniasis, Leishmania infantum, biomarkers, cytokines/chemokines, canine visceral leishmaniasis, human visceral leishmaniasis

\section{INTRODUCTION}

Leishmaniasis is considered a neglected tropical disease with approximately 350 million people at risk of infection, and with 2 million new cases reported annually, mainly in extremely impoverished communities (WHO/Leishmaniasis, 2014). The clinical manifestations of leishmaniasis range from cutaneous ulcers to the visceral form, one of the most severe which can be fatal if left untreated (Desjeux, 2004). Over $90 \%$ of visceral leishmaniasis (VL) cases worldwide are concentrated in six countries: India, Bangladesh, Sudan, South Sudan, Ethiopia, and Brazil. VL arises from either $L$. donovani in the Indian subcontinent and East Africa, or L. infantum in Europe and Latin America, and kills 20,000-40,000 people annually throughout the world (Alvar et al., 2012).

Dogs are considered the main reservoirs of $L$. infantum parasites and the presence of these animals in endemic areas represents a risk factor for human disease. The disease in dogs shares characterisitcs with human VL, providing a good model to study the immunopathogenesis of $L$. infantum infections. Canine visceral leishmaniasis (CVL) is also of veterinary interest since the disease is spreading to big cities in Latin America including Belo Horizonte, São Paulo, Natal, and Camaçari (Alves and Bevilacqua, 2004), threatening public health. 
L. infantum multiplies inside macrophages in the liver, spleen and bone marrow. In human VL, 90\% of infected individuals remain asymptomatic or subclinical, showing an intense cellular immune response characterized by a positive delayed-type hypersensitivity reaction to Leishmania antigens. However, in patients who progress to clinical disease, an enlargement of the spleen and liver may be observed, accompanied by hematological disorders, notably anemia, thrombocytopenia, which may result in hemorrhaging, and neutropenia. These disorders can increase host susceptibility to bacterial infection and patients with VL often suffer weight loss or fever (Werneck et al., 2003).

The search for biomarkers for prognosis of human and canine $\mathrm{VL}$ and measurement of the success of treatment has intensified in the last few years. This led to a better knowledge of disease immunopathogenesis and favored therapeutic and prophylactic strategies for ZVL. As L. infantum is transmitted by the bite of phlebotomine sand flies, several markers of vector exposure have also been identified and used as tools to assess success of interventions. Used in combination, biomarkers of disease and vector exposure can provide powerful tools to support efforts to control ZVL.

\section{HUMAN ZVL}

The progression of ZVL is associated with immunosuppression, characterized by lack of a cell-mediated immune response to Leishmania antigens. Accordingly, the absence of lymphocyte blastogenesis and IFN- $\gamma$ production have been associated with progression to VL (Carvalho et al., 1992). Interestingly, patients become responsive to Leishmania antigens after successful therapy (Carvalho et al., 1989). Biomarkers were evaluated in a group of ZVL patients given standard antimonial treatment (Schriefer et al., 1995). Soluble CD4 (sCD4), sCD8, and sIL-2R levels were higher in sera of patients compared to healthy controls. After treatment, levels of the above-mentioned biomarkers exhibited a significant decrease in patients who responded to therapy. Importantly, when comparing pretreatment levels of these markers among those who responded to antimonial therapy and refractory patients, the serum concentrations of $\mathrm{SCD} 8, \mathrm{sIL}-2 \mathrm{R}$ as well as neopterin were significantly elevated in refractory patients. Therefore, these markers represent promising indicators of a patient's response to antimonial therapy (Schriefer et al., 1995).

The cure for human ZVL is associated to induction of a Th1 immune response characterized by IFN- $\gamma$ production. IFN$\gamma$ has an essential role in controlling the parasite load and in the development of a long-lasting immunity. Asymptomatic patients also exhibit a Th1 response, suggesting that IFN- $\gamma$ activates macrophages, increasing their leishmanicidal ability and maintaining the infection under control (Kaye and Scott, 2011). In contrast, anti-inflammatory cytokines, mainly IL-10, lead to proliferation of parasites and interfere with infection control (Nylen and Sacks, 2007; Gautam et al., 2011). In a study of patients with active ZVL, in vitro stimulation of PBMC with Leishmania antigens showed an inverse pattern with low levels of IFN- $\gamma$ during active disease that augmented steadily after treatment (Caldas et al., 2005). Interestingly, these patients showed elevated plasma levels of IFN- $\gamma$, IL-12p40, and IL-10 during active disease that sharply decreased after treatment (Caldas et al., 2005). Therefore, IFN- $\gamma$ and IL-10 are the main hallmarks of infection by L. infantum, and the balance between these cytokines seems to be essential for control of the infection.

IL-17, a cytokine produced mainly by Th17 cells, is known for inducing the production of chemokines that recruit neutrophils to inflammatory sites. A cohort of individuals with VL caused by $L$. donovani showed that IL-17 seems to be protective (Pitta et al., 2009). However, in ZVL patients caused by L. infantum, high levels of this cytokine did not induce IFN $\gamma / \mathrm{NO}$ in enough concentrations to lead to a recovery from disease (Nascimento et al., 2015).

In severe forms of ZVL, there is an exaggerated inflammatory response that leads to disseminated intravascular coagulation and other manifestations such as hemorrhage (Costa et al., 2013). Children displaying an intense production of cytokines have a higher risk of death. IL- 6 seems to be one of the main cytokines associated with fatal disease, but IFN- $\gamma$, IL- $1 \beta$, IL-8, and TNF- $\alpha$ have also been associated to ZVL severity (Costa et al., 2013). In fact, $L$. infantum may activate inflammatory reactions via CD14, leading to a production of several cytokines such as IFN- $\gamma$, IL27, IL-10, IL-6 as well as sCD14 (Dos Santos et al., 2016). These data reinforce previous results and denote the interdependent relationship between pro-inflammatory (IFN- $\gamma$, IL- 6 and TNF- $\alpha$ ) and anti-inflammatory (IL-10 and IL27) cytokines. The authors also showed that higher levels of IL-6 (>200 pg/ml) are associated to death (Dos Santos et al., 2016). Collectively, this highlights the complexity of finding a good biomarker for ZVL since induction of a cytokine such as IFN- $\gamma$ may be associated to protection or severe disease, depending on its levels and the overall inflammatory environment.

Other biomarkers, including lipid mediators, have been associated with ZVL and could also be used to monitor the efficacy of specific therapies. Araujo-Santos et al. (2017) reported a distinct biosignature of active ZVL through increased serum levels of Prostaglandin F 2 alfa $\left(\mathrm{PGF}_{2 \alpha}\right)$, Leukotrine B4 $\left(\mathrm{LTB}_{4}\right)$, Resolvin D1 (RvD1), TNF- $\alpha$, IL-1 $\beta$, IL-6 and IL-8, IL-10, and IL-12p70, as well as decreased concentrations of TGF$\beta 1$ in comparison to healthy endemic controls, regardless of patient age or gender. Following the onset of leishmanicidal treatment, the inflammatory cytokine profile, as well as the relationships between these markers and several hematological and biochemical parameters, gradually reverted, which suggested that the observed cytokine expression profile was induced by active disease or infection (Araujo-Santos et al., 2017). Of the quantified markers, TGF- $\beta 1$ concentrations were significantly elevated, while IL-6, IL-8, IL-10, and RvD1 levels substantially decreased, after 30 days of therapy in comparison to their levels during active ZVL infection.

It is worthwhile noting that common immunological signatures were observed in sera of VL patients from Brazil and Bangladesh infected with L. infantum and L. donovani, respectively. Inflammatory and regulatory cytokines (IFN $\gamma$, 
TNF $\alpha$, IL-10, IL-17), as well as levels of growth factors (FGFfibroblast growth factor; VEGF, vascular endothelial growth factor), were elevated in the serum of VL patients from both regions (Duthie et al., 2014). Serological assays from Brazilian patients obtained during and after meglumine antimoniate treatment demonstrated that multiple parameters reverted to concentrations similar to healthy endemic controls. The authors suggested that a multi-parameter signature of the response to treatment could be useful in clinical trials to evaluate the success of therapeutic interventions (Duthie et al., 2014).

Macrophages infected by $L$. infantum present an M2b-like phenotype as well as a C-type lectin receptor (CLR) signature, characterized by Dectin-1, mannose receptor and DC-SIGN homolog SIGNR3 expression (Lefevre et al., 2013). Expression of Dectin-1 and the mannose receptor are essential to the leishmanicidal effect of macrophages, leading to the production of ROS and also the induction of IL- $1 \beta$ secretion. On the other hand, SIGNR3 was shown to favor parasite survival via the inhibition of the LTB-4-IL1 $\beta$ axis (Lefevre et al., 2013).

More recently, MCP-1 was shown to be a good biomarker to identify asymptomatic individuals infected by L. infantum (Ibarra-Meneses et al., 2017). This chemokine is expressed 110 times more strongly than IL-2 in cultures of whole blood stimulated with Leishmania antigens, identifying $87.5 \%$ of asymptomatic subjects; it is also significantly increased in all patients cured of ZVL (Ibarra-Meneses et al., 2017). Table 1 summarizes the main findings about biomarkers in human ZVL.

In summary, biomarkers other than IFN- $\gamma$ and IL-10 have been described more recently that reveal the complexity of ZVL. These molecules have demonstrated both their value as biomarkers of disease progression and their usefulness in monitoring the efficacy of treatment. In the future, such biomarkers may also be of value in assessing the level of protection induced by prophylactic strategies.

\section{CANINE VL}

Dogs are one of the main urban reservoirs of $L$. infantum parasites and their presence in endemic areas is a risk factor for the development of human disease, due to their role in propagating infection in phlebotomine sand flies. Clinical manifestations of CVL present a wide spectrum of clinical signs that are non-specific. However, a high proportion of animals do not progress to disease, control the parasites and live for years or their entire life without any clinical signs (Foglia Manzillo et al., 2013). The presence of these dogs in the endemic area contributes to maintenance of the parasites, since they can transmit $L$. infantum to the sand flies. The resistance or susceptibility to CVL is directly correlated with the induction of either a Th1 response characterized by IFN- $\gamma$, IL-2, and TNF- $\alpha$ production, or a Th2 response with the production of IL-4, IL-5, IL-10, IL-13, and TGF- $\beta$, respectively, and the level of immune activation is considered to directly influence disease severity (Reis et al., 2010; Barbosa et al., 2011). In fact, a reduction in the burden of $L$. infantum was related to elevated expression of IFN- $\gamma$ and TNF- $\alpha$, whereas increased IL-10 and iron regulatory protein 2 (IRP2) expression and an increase in plasma albumin levels were associated with a higher parasite burden (do Nascimento et al., 2013). Accordingly, the dynamics between different aspects of the immune response and intracellular iron availability could play some role in the evolution of Leishmania infection (do Nascimento et al., 2013).

TABLE 1 | Biomarkers for human visceral leishmaniasis.

\begin{tabular}{|c|c|c|c|c|c|}
\hline Cytokines & Chemokine & Lipid mediators & Mixed molecules & Signature & References \\
\hline \multirow[t]{4}{*}{$\begin{array}{l}\text { IFN- } \gamma, \text { IL-12, and } \\
\text { IL- } 10 \text { TNF } \alpha, \text { IL-17 }\end{array}$} & & & $\begin{array}{l}\text { sCD4, sCD8, } \\
\text { and sIL2R }\end{array}$ & $\uparrow$ Active disease & $\begin{array}{l}\text { Schriefer et al., 1995; } \\
\text { Gautam et al., } 2011\end{array}$ \\
\hline & & & & $\downarrow$ Post-treatment & $\begin{array}{l}\text { Nylen and Sacks, 2007; } \\
\text { Duthie et al., 2014; } \\
\text { Araujo-Santos et al., } 2017\end{array}$ \\
\hline & & & $\begin{array}{l}\text { sCD8, sIL-2R, } \\
\text { neopterin }\end{array}$ & $\begin{array}{l}\uparrow \text { Patients refractory to } \\
\text { treatment }\end{array}$ & Caldas et al., 2005 \\
\hline & MCP-1 & & & $\begin{array}{l}\uparrow \text { Asymptomatic VL } \\
\uparrow \text { Cured patients }\end{array}$ & $\begin{array}{l}\text { Kaye and Scott, 2011; } \\
\text { Ibarra-Meneses et al., } 2017\end{array}$ \\
\hline IL-6 & & & & $\begin{array}{l}\uparrow \text { Fatal Disease (higher risk } \\
\text { of death) }\end{array}$ & $\begin{array}{l}\text { Costa et al., 2013; Dos } \\
\text { Santos et al., } 2016\end{array}$ \\
\hline \multirow{2}{*}{$\begin{array}{l}\text { IL-1 } \beta, \text { IL-8, and } \\
\text { TNF- } \alpha, \text { IFN- } \gamma \text {, } \\
\text { IL-27, IL-10 }\end{array}$} & & & & Severity of disease & Dos Santos et al., 2016 \\
\hline & & & sCD14 & $\uparrow$ Severe disease & Dos Santos et al., 2016 \\
\hline $\begin{array}{l}\text { IL-1 } \beta, \text { IL-6 IL-8 } \\
\text { TGF- } \beta 1\end{array}$ & & $\begin{array}{l}\text { PGF2 } \alpha, \text { LTB4, } \\
\text { RvD1 }\end{array}$ & & $\begin{array}{l}\downarrow \text { Active ZVL Post-treatment } \\
\downarrow \text { Active ZVL } \\
\downarrow \text { Post-treatment }\end{array}$ & Araujo-Santos et al., 2017 \\
\hline- & & & FGF, VEGF & $\begin{array}{l}\uparrow \text { Active VL } \\
\downarrow \text { Post-treatment }\end{array}$ & Duthie et al., 2014 \\
\hline
\end{tabular}


The predominance of a Th2 response in infected dogs leads to the appearance of M2 macrophages, identified by CD163 immunostaining, mainly in the spleen, muzzle, ear and popliteal and pre-capsular lymph nodes (Moreira et al., 2016). The highest proportion of M2 macrophages coincided with the highest parasite loads and were found in more susceptible organs of infected dogs such as the spleen and lymph nodes, as well as skin, considered a more resistant organ. In contrast, the liver showed low parasitism and weak immunostaining for M2 macrophages that was not significantly different between infected and negative groups of dogs. Therefore, M2 macrophages may contribute to parasite proliferation in organs (Moreira et al., 2016). Another important point that contributes to the gravity of CVL is associated with the disorganization of splenic tissue. Dogs that presented positive spleen cultures also showed more disrupted spleen architecture, with a lower concentration of serum albumin and creatinine and higher levels of aspartate aminotransferase. Together, these data suggest that the disorganization of lymphoid tissue in the spleen is linked to more severe clinical presentations of CVL (Lima et al., 2014).

The immunosuppression observed during CVL is also correlated to the occurrence of $\mathrm{CD} 4+$ and $\mathrm{CD} 8+\mathrm{T}$ cell exhaustion and could be responsible for the absence of specific antigen blastogenesis and IFN- $\gamma$ secretion. Moreover, there was a significant increase in the surface expression of programmed death 1 (PD-1) on $\mathrm{T}$ cell populations, mainly in CD8 $\mathrm{T}$ cells of symptomatic dogs in comparison to control animals. Using monoclonal antibodies able to inhibit the PD-1 ligand B7.H1 restored $\mathrm{CD}^{+}$and $\mathrm{CD} 8^{+} \mathrm{T}$ cell function and raised the levels of reactive oxygen species in cocultures of phagocytes and $\mathrm{T}$ cells. Consequently, these macrophages showed a reduction in the number of intracellular parasites. The $\mathrm{T}$ cell exhaustion resulting from symptomatic CVL may affect the response to vaccination and the efficacy of treatments used to control L. infantum (Esch et al., 2013).

Different reports in the literature have associated the parasite burden in different tissues with the host immune response, evaluating pro- and anti-inflammatory cytokines. Generally, there is a consensus that a mixed Th1/ Th2 response is observed at the time of detection of a Leishmania infection, despite the prevalence of a Th2 profile. Later in the infection, when the parasite load decreases, e.g., as a result of treatment, the Th1 profile becomes predominant. However, this is not always the case. In a study of 20 infected dogs treated with miltefosine and allopurinol, $80 \%$ of animals showed expansion of the parasite load in blood and lymph nodes and low IFN$\gamma$ levels at the end of the 9-12 months study period (Manna et al., 2008). This response signals a failure to induce a Th1 response in the majority of treated dogs. Nevertheless, these animals did not show any sign of disease, suggesting they are in an asymptomatic state (Manna et al., 2008). The above highlights the complexity of CVL and the absence of reliable markers of recovery of infected dogs. In another study, quantifying TNF- $\alpha$, IL-4, and IL-10 in the spleen and liver of dogs naturally infected with $L$. infantum, with or without clinical signs, showed that the animals exhibited higher levels of these cytokines compared to control non-infected dogs (DE F Michelin et al., 2011). Interestingly, the authors found that the liver is the main organ responsible for the production of cytokines during infection. Moreover, TNF- $\alpha$ was positively correlated with parasite burden and could represent a marker for disease infection, with the participation of IL-10 (DE F Michelin et al., 2011).

More recently, the parasite burden and levels of IFN- $\gamma$, TNF- $\alpha$, IL-10, and TGF- $\beta$ were evaluated in 5 target tissues at 6 and 16 months after infection with $L$. infantum in an experimental canine model (Rodriguez-Cortes et al., 2016). The data showed that the spleen and liver of infected animals exhibited a high parasite density at both time points and produced pro- and anti-inflammatory responses (RodriguezCortes et al., 2016). The popliteal lymph nodes produced IFN$\gamma$ both at the beginning of the infection and in the chronic phase. In contrast, an increase in IL-10 and TGF- $\beta$ expression in these organs was only observed in the chronic phase. Of note, cytokines were absent in the skin, although parasites were detected at 6 months post-infection. Therefore, in the above-mentioned study, the spleen and liver of infected dogs produced diverse cytokines at early times of infection, whereas an anti-inflammatory profile was observed in peripheral tissues at later periods, considered as the chronic phase of infection (Rodriguez-Cortes et al., 2016). Another study of experimentally infected dogs, considered asymptomatic due to the few clinical signs presented, animals were maintained for six years after intradermal infection (Abbehusen et al., 2017). Although few clinical signs were noticed in these animals, most presented parasites in the lymph nodes, spleen and skin and exhibited an increase in IFN- $\gamma$, GM-CSF, IL-6, and IL-18 levels and a decrease in TNF, IL-2, and CXCL1 serum concentrations. These results seem to suggest that a persistent activation of the immune system in subclinical infections with $L$. infantum may possibly control parasite growth and limit disease severity (Abbehusen et al., 2017).

Our group studied 70 naturally infected dogs from an endemic area in Bahia, Brazil, grouping the animals according to a clinical score previously described by Manna et al (Manna et al., 2009) with slight modifications. In the group of dogs with severe disease (clinical score $>7$ ), we observed a reduction in the levels of serum LTB-4 and PGE-2 and an elevation in chemokine concentrations such as CXCL1 and CCL2 (Solca et al., 2016). Performing ROC curves, we observed that a combination of LTB-4, PGE-2, and CXCL-1 differentiated best among distinct groups of dogs with different clinical scores. Besides that, analysis of the interactome of the different mediators evaluated showed that LTB-4, a lipid mediator, had the highest number of interactions with other cytokines and chemokines in the group of dogs with severe disease. Although IFN- $\gamma$ and IL-10 were also evaluated, we did not find an important role for these cytokines as markers of disease progression in infected dogs (Solca et al., 2016). Table 2 summarizes the main findings about biomarkers in canine ZVL.

In CVL, the complexity of the immune response and the role played by different mediators during infection by $L$. infantum is clear. Although IFN- $\gamma$ and IL-10 seem to be important cytokines 
TABLE 2 | Biomarkers in canine visceral leishmaniasis.

\begin{tabular}{|c|c|c|c|c|c|}
\hline Cytokines & Chemokines & $\begin{array}{l}\text { Lipid } \\
\text { mediators }\end{array}$ & Mixed molecules & Signature & References \\
\hline \multirow[t]{2}{*}{$\begin{array}{l}\text { IFN- } \gamma, \mathrm{IL}-2 \\
\text { and TNF- } \alpha\end{array}$} & & & & $\uparrow$ Resistance to infection & $\begin{array}{l}\text { Reis et al., 2010; Barbosa } \\
\text { et al., 2011; do Nascimento } \\
\text { et al., } 2013\end{array}$ \\
\hline & & & & $\uparrow$ Beginning of infection & $\begin{array}{l}\text { Rodriguez-Cortes et al., } \\
2016\end{array}$ \\
\hline \multirow[t]{2}{*}{ TGF- $\beta$} & & & & $\uparrow$ Chronic phase & $\begin{array}{l}\text { Rodriguez-Cortes et al., } \\
2016\end{array}$ \\
\hline & & & $\begin{array}{l}\text { IRP2 (iron } \\
\text { regulatory } \\
\text { protein 2) }\end{array}$ & $\uparrow$ Susceptible dogs & do Nascimento et al., 2013 \\
\hline $\begin{array}{l}\text { IFN- } \gamma \text {, GM-CSF, } \\
\mathrm{IL}-6 \text {, and IL-18 }\end{array}$ & & & & $\begin{array}{l}\uparrow \text { Dogs infected by } 6 \text { years } \\
\text { without clinical signs }\end{array}$ & Abbehusen et al., 2017 \\
\hline \multirow[t]{3}{*}{ TNF, IL-2 } & CXCL1 & & & $\begin{array}{l}\downarrow \text { Dogs infected by } 6 \text { years } \\
\text { without clinical signs }\end{array}$ & \\
\hline & & $\begin{array}{l}\text { LTB-4 and } \\
\text { PGE-2 }\end{array}$ & & $\begin{array}{l}\downarrow \\
\text { Dogs with severe disease }\end{array}$ & Solca et al., 2016 \\
\hline & $\begin{array}{l}\text { CCL2, } \\
\text { CCXL1 }\end{array}$ & & & $\uparrow$ & \\
\hline
\end{tabular}

and are induced during infection, other mediators could be more useful as markers of disease progression or in the assessment of the response to treatment. An important point that deserves consideration is the presence of parasites in different organs of infected dogs, including skin. As such, asymptomatic dogs could represent a good source of parasites to uninfected sand flies contributing to their persistence in endemic areas. These findings indicate that the search for markers of exposure to sand flies is essential for surveillance of endemic areas.

\section{BIOMARKERS OF VECTOR EXPOSURE}

In large part, "biomarkers" is a term mostly used to describe characteristics of a disease. However, for vector-borne diseases they can also reference exposure to the arthropod vectors that transmit them. This relatively new discipline of research has gained momentum in recent years and is proving to be of value to various approaches aiming at disease treatment or control (Andrade and Teixeira, 2012; Doucoure and Drame, 2015; Lestinova et al., 2017). In contrast to disease biomarkers, good vector exposure biomarkers may be used as preventative tools that can identify a heightened risk for contracting disease (Carvalho et al., 2015; Ya-Umphan et al., 2017). Further, good biomarkers of exposure may also be used to monitor the expansion or contraction of vector ranges over time, an important tool in light of climate change and its effect on changing the global distribution of disease vectors (Parham et al., 2015; Purse et al., 2015).

The basic requirements that define a good disease biomarker also apply to biomarkers of vector exposure. A reliable biomarker should be specific to a particular vector species (Poinsignon et al., 2008; Teixeira et al., 2010; Ali et al., 2012; Doucoure et al., 2012; Zhao et al., 2015), and recognized by the majority of the exposed or target host population (Souza et al., 2010; Marzouki et al., 2012; Doucoure and Drame, 2015; Mukbel et al., 2016). Additionally, an ideal vector biomarker should be relatively short-lived in the absence of exposure, a desired feature for use in biomonitoring (Clements et al., 2010; Gidwani et al., 2011; Noukpo et al., 2016). For arthropods that transmit pathogens by bite, which represents the majority of disease vectors including phlebotomine sand flies, saliva has been the primary target for biomarkers of vector exposure (Andrade and Teixeira, 2012; Courtin et al., 2015; Doucoure and Drame, 2015).

Most vectors bite mammalian hosts to acquire blood. As such, saliva of vectors has evolved to facilitate blood feeding (Ribeiro and Francischetti, 2003; Lestinova et al., 2017) However, in addition to their physiological effects, several salivary molecules of major disease vectors are immunogenic in humans as well as animal reservoirs and have the potential to become biomarkers of exposure. (Andrade and Teixeira, 2012; Dama et al., 2013a; Abdeladhim et al., 2014; Doucoure and Drame, 
2015). Considering that vector control continues to be considered one of the most effective methods to inhibit the transmission of vector-borne diseases, developing immunogenic salivary proteins as biomarkers of vector exposure represent a powerful tool in our arsenal toward their control.

Measuring the antibody response to total saliva, though useful, has serious limitations, mostly due to cross-reactivity of some antigens in various sympatric vectors or host-biting non-vectors (Andrade and Teixeira, 2012; Dama et al., 2013a,b; Doucoure and Drame, 2015; Simo et al., 2017). There are also technical limitations to the use of total saliva preparations as marker of vector exposure including reproducibility and scale-up. This prohibits its consideration for use in largescale epidemiological surveys. To overcome such obstacles, well-defined salivary antigens or peptides that are specific to a particular vector species, while maintaining a measurable immunogenicity in the target population, are being pursued (Rohousova et al., 2005; Poinsignon et al., 2008; Teixeira et al., 2010; Ali et al., 2012; Zhao et al., 2015; Sima et al., 2016)

\section{Biomarkers of Exposure to Vector Sand Flies}

Sand fly saliva consists of a relatively small number of secreted proteins that are injected into the skin in small quantities (Abdeladhim et al., 2014). Nevertheless, sand fly saliva is highly immunogenic and induces a potent cellular and humoral immune response in humans and animals including dogs, known reservoirs of L. infantum (Andrade and Teixeira, 2012; Abdeladhim et al., 2014; Lestinova et al., 2017). Though it has been well established that induction of a saliva-specific Th1-biased cellular immunity protects from disease, salivaspecific antibodies have been associated to both protection, for vectors of visceral leishmaniasis (Barral et al., 2000; Gomes et al., 2002; Aquino et al., 2010; Vlkova et al., 2011), as well as an enhanced risk of infection, for vectors of cutaneous leishmaniasis (Mondragon-Shem et al., 2015). To date, we have no clear understanding of the reason behind these contrasting associations.

Whether antibodies to saliva are associated to protection or risk, they remain a good indicator of the rate of exposure to bites of vector sand flies. However, as for other vectorborne diseases, there are more than one man-biting species of sand flies in endemic areas, and sympatric vectors transmitting different types of leishmaniasis are not uncommon (Rohousova et al., 2005; Clements et al., 2010; Teixeira et al., 2010; Marzouki et al., 2012). This increases the risk of cross-reactive antigens between different species of sand flies and decreases from the efficacy of total saliva as reliable biomarkers of vector exposure. For this reason, defined salivary antigens specific to a particular vector species are being developed as markers of exposure in humans and reservoirs (Andrade and Teixeira, 2012; Lestinova et al., 2017). One of the most promising markers of human exposure to vector sand flies is PpSP32, a salivary protein from Phlebotomus papatasi. PpSP32 was identified as an immunodominant antigen in a naturally exposed population in Tunisia and was later validated in largescale studies of endemic populations in Tunisia and Saudi Arabia (Marzouki et al., 2012, 2015; Mondragon-Shem et al., 2015).

\section{Biomarkers of Exposure to Bites of Lutzomyia longipalpis, the Principal Vector of ZVL in Latin America}

Lutzomyia longipalpis, the main vector of ZVL in Latin America, has a wide distribution range extending from Mexico to Uruguay (Lainson and Rangel, 2005; Brazil, 2013). The domestic dog is considered a main reservoir host and is a major source of sand fly infection and human disease (Lainson and Rangel, 2005; Roque and Jansen, 2014). As such, it is clear that developing reliable biomarkers of exposure to $L$. longipalpis for humans and dogs would provide a useful epidemiological tool for monitoring ZVL in Latin America. Two studies have demonstrated the immunogenicity of L. longipalpis saliva in endemic populations, associating a positive saliva-specific antibody response to protection against ZVL (Barral et al., 2000; Aquino et al., 2010). The screening of recombinant proteins representing major secreted salivary molecules in L. longipalpis saliva revealed LJM11 and LJM17 as potential markers of vector exposure for both humans and dogs (Teixeira et al., 2010). Both LJM11 and LJM17 belong to the yellow family of salivary proteins that bind biogenic amines blocking their hemostatic-restoring activity during feeding (Abdeladhim et al., 2014). Relevant to their function as biomarkers of exposure, LJM11 and LJM17 are abundant in sand fly saliva and absent from saliva of other common insects such as mosquitoes (Abdeladhim et al., 2014). However, for use as specific markers of exposure to L. longipalpis saliva, absence of cross-reactivity with their homologs in saliva of other sympatric sand fly species needs to be demonstrated. Both LJM11 and LJM17 are not recognized by sera of humans bitten by L. intermedia, a vector of cutaneous leishmaniasis in Brazil whose distribution commonly overlaps with L. longipalpis (Teixeira et al., 2010). Further, only one of two human sera that are strongly reactive to $L$. longipalpis saliva weakly recognized two antigens in $L$. intermedia saliva (Teixeira et al., 2010). A large-scale study further validated the immunogenicity and specificity of LJM11 and LJM17 as markers of exposure to L. longipalpis saliva (Souza et al., 2010). Moreover, sensitivity of the assay was enhanced to a level comparable to that against total saliva by using a combination of both LJM11 and LJM17 (Souza et al., 2010).

In contrast to other zoonotic Leishmania infections, canids are considered the only reservoir of $L$. infantum infection. As such, developing a marker of vector exposure to L. longipalpis for dogs is indicated. LJM11 and LJM17 and two other salivary proteins from L. longipalpis saliva, LJL143 and LJL23, were highly immunogenic in dogs (Collin et al., 2009; Teixeira et al., 2010). LJL143, also called Lufaxin, has dual anticoagulant and anti-complement activities (Collin et al., 2012; Mendes-Sousa et al., 2017), while LJL23 is an Apyrase, an inhibitor of platelet aggregation (Teixeira et al., 2010; Abdeladhim et al., 2014). 
Interestingly, SP01B and SP01, Apyrase homologs from saliva of $P$. perniciosus, one of the primary vectors of $L$. infantum in Europe, were also recognized as markers capable of detecting vector exposure in dogs (Abdeladhim et al., 2014; Lestinova et al., 2017) Moreover, SP03B, a yellow protein from P. perniciosus saliva was also identified as a good marker of vector exposure in dogs (Kostalova et al., 2017). However, to our knowledge, the specificity of SP01B, SP01, and SP03B to saliva of P. perniciosus have not been tested in areas where other dog-biting sand fly species are prevalent. Though geographically separated, it is interesting to note that molecules belonging to the yellow family of proteins and Apyrases of both L. longipalpis and $P$. pernicious were identified as promising candidates for vector exposure to ZVL in both humans and dogs (Teixeira et al., 2010; Lestinova et al., 2017). In addition to their use to assess human and dog exposure to sand fly bites, biomarkers can also be developed for use in sentinel animals that are common around humans, such as chicken or domestic ungulates, as indicators of vector prevalence (Soares et al., 2013; Rohousova et al., 2015).

The above-mentioned defined biomarkers of L. longipalpis bites are highly promising, however, considering the richness of the sand fly fauna in Latin America, they require further validation of their specificity against salivary homologs from other dominant man- or dog-biting sand fly species. Having said that, the level of specificity of a biomarker should be primarily indicated by its intended use and by the nature of the endemic area. A biomarker can target a particular vector, several vectors, or simply sand fly bites.

Another significant utility for biomarkers of vector exposure is biomonitoring. For this, knowledge of the kinetics of antibody induction and decay is necessary. Previous studies in humans and dogs have demonstrated that saliva-specific antibodies correlate to biting intensity, fluctuate with the sand fly season and are of relatively short duration, declining significantly after bite cessation caused by sand fly seasonality, use of nets or removal from endemic areas, thus demonstrating their usefulness as tools to measure efficacy of vector control interventions (Hostomska et al., 2008; Clements et al., 2010; Gidwani et al., 2011; Vlkova et al., 2011; Marzouki et al., 2012; Kostalova et al., 2015; Quinnell et al., 2018). Nevertheless, the antibody response to saliva may be species-specific and may change in response to a defined antigen. Therefore, kinetics and duration of antibodies should be investigated for the sand fly vector species/biomarker in question. For L. longipalpis, two studies investigated the kinetics of antibody response to total saliva in dogs experimentally (Hostomska et al., 2008) or naturally (Quinnell et al., 2018) exposed to bites. In experimentallyexposed dogs, the level of saliva-specific total IgG antibodies correlated positively to the intensity of bites, increasing rapidly with each weekly exposure and declining significantly within 2 weeks of the last exposure, despite maintaining a low titer up to 6 months post-exposure (Hostomska et al., 2008). In dogs naturally exposed to L. longipalpis bites, dogs developed salivaspecific antibodies in 2 months, with antibody levels increasing during high transmission/biting intensity and declining rapidly during low transmission/biting intensity (Quinnell et al., 2018).

To date, we have developed the methodologies that identified several promising makers of exposure to L. longipalpis bites. Further studies of the utility of these defined antigens, as biomarkers of exposure to L. longipalpis need to be undertaken in natural foci to thoroughly investigate their specificity. Additionally, longitudinal studies to establish the kinetics of antibody development and decline to promising defined biomarkers will establish their value in biomonitoring and may even reveal important associations with risk of, or protection from, ZVL.

\section{OVERALL CONCLUSION}

The complexity of ZVL and CVL challenges the reliability of a single biomarker to assess disease progression in humans and dogs, respectively. More likely, a combination of distinct inflammatory mediators will be needed to provide a tool that can distinguish relevant states of disease, also defining the role played by these different molecules in the pathogenesis of ZVL.

Despite the considerable progress made in defining important biomarkers for both ZVL and CVL, more studies are indicated, as well as an open dialogue by the scientific community, to reach a consensus for a reliable signature of distinct disease states. Another important point to consider evaluating the different biomarkers in ZVL is the possibility to find new targets to improve the treatment of the disease, increasing the chances of cure and avoiding the fatal outcome of infection. The combination of different drugs directed to several molecules would contribute to obtain an effective treatment for patients. In addition, in the future, such biomarkers may also be of value in assessing the level of protection induced by prophylactic strategies.

Together with well-defined markers of exposure to vector sand flies, such tools could become invaluable to evaluate response to treatment, and success of interventions among others.

\section{AUTHOR CONTRIBUTIONS}

$\mathrm{CB}$ and SK have equal participation to write this review.

\section{FUNDING}

This work was partly supported by the Intramural Research Program of the NIH, National Institute of Allergy and Infectious Diseases and by FAPESB (Fundação de Apoio a Pesquisa do Estado da Bahia), grant numbers SUS0036/2013 and PET0024/2013. CB is a senior investigator of the National Council of Research (CNPq).

\section{ACKNOWLEDGMENTS}

The authors thank Andrezza Kariny and Juliana Oliveira for secretarial assistance. 


\section{REFERENCES}

Abbehusen, M. M. C., Almeida, V. D. A., Solca, M. D. S., Pereira, L. D. S., Costa, D. J., Gil-Santana, L., et al. (2017). Clinical and immunopathological findings during long term follow-up in Leishmania infantum experimentally infected dogs. Sci. Rep. 7:15914. doi: 10.1038/s41598-017-15651-8

Abdeladhim, M., Kamhawi, S., and Valenzuela, J. G. (2014). What's behind a sand fly bite? The profound effect of sand fly saliva on host hemostasis, inflammation and immunity. Infect. Genet. Evol. 28, 691-703. doi: 10.1016/j.meegid.2014.07.028

Ali, Z. M., Bakli, M., Fontaine, A., Bakkali, N., Vu Hai, V., Audebert, S., et al. (2012). Assessment of Anopheles salivary antigens as individual exposure biomarkers to species-specific malaria vector bites. Malar. J. 11:439. doi: 10.1186/1475-2875-11-439

Alvar, J., Velez, I. D., Bern, C., Herrero, M., Desjeux, P., Cano, J., et al. (2012). Leishmaniasis worldwide and global estimates of its incidence. PLoS ONE 7:e35671. doi: 10.1371/journal.pone.0035671

Alves, W. A., and Bevilacqua, P. D. (2004). Quality of diagnosis of canine visceral Leishmaniasis in epidemiological surveys: an epidemic in Belo Horizonte, Minas Gerais, Brazil, 1993-1997. Cad. Saude Publ. 20, 259-265. doi: 10.1590/S0102-311X2004000100043

Andrade, B. B., and Teixeira, C. R. (2012). Biomarkers for exposure to sand flies bites as tools to aid control of Leishmaniasis. Front. Immunol. 3:121. doi: 10.3389/fimmu.2012.00121

Aquino, D. M., Caldas, A. J., Miranda, J. C., Silva, A. A., Barral-Netto, M., and Barral, A. (2010). Epidemiological study of the association between anti-Lutzomyia longipalpis saliva antibodies and development of delayed-type hypersensitivity to Leishmania antigen. Am. J. Trop. Med. Hyg. 83, 825-827. doi: 10.4269/ajtmh.2010.10-0182

Araujo-Santos, T., Andrade, B. B., Gil-Santana, L., Luz, N. F., Dos Santos, P. L., de Oliveira, F. A., et al. (2017). Anti-parasite therapy drives changes in human visceral Leishmaniasis-associated inflammatory balance. Sci. Rep. 7:4334. doi: 10.1038/s41598-017-04595-8

Barbosa, M. A., Alexandre-Pires, G., Soares-Clemente, M., Marques, C., Rodrigues, O. R., De Brito, T. V., et al. (2011). Cytokine gene expression in the tissues of dogs infected by Leishmania infantum. J. Comp. Pathol. 145, 336-344. doi: 10.1016/j.jcpa.2011.03.001

Barral, A., Honda, E., Caldas, A., Costa, J., Vinhas, V., Rowton, E. D., et al. (2000). Human immune response to sand fly salivary gland antigens: a useful epidemiological marker? Am. J. Trop. Med. Hyg. 62, 740-745.

Brazil, R. P. (2013). The dispersion of Lutzomyia longipalpis in urban areas. Rev. Soc. Bras. Med. Trop. 46, 263-264. doi: 10.1590/0037-8682-0101-2013

Caldas, A., Favali, C., Aquino, D., Vinhas, V., van Weyenbergh, J., Brodskyn, C., et al. (2005). Balance of IL-10 and interferon-gamma plasma levels in human visceral Leishmaniasis: implications in the pathogenesis. BMC Infect. Dis. 5:113. doi: 10.1186/1471-2334-5-113

Carvalho, A. M., Cristal, J. R., Muniz, A. C., Carvalho, L. P., Gomes, R., Miranda, J. C., et al. (2015). Interleukin 10-dominant immune response and increased risk of cutaneous leishmaniasis after natural exposure to lutzomyia intermedia sand flies. J. Infect. Dis. 212, 157-165. doi: 10.1093/infdis/jiv020

Carvalho, E. M., Bacellar, O., Barral, A., Badaro, R., and Johnson, W. D. Jr. (1989). Antigen-specific immunosuppression in visceral Leishmaniasis is cell mediated. J. Clin. Invest. 83, 860-864. doi: 10.1172/JCI113969

Carvalho, E. M., Barral, A., Pedral-Sampaio, D., Barral-Netto, M., Badaro, R., Rocha, H., et al. (1992). Immunologic markers of clinical evolution in children recently infected with Leishmania donovani chagasi. J. Infect. Dis. 165, 535-540. doi: 10.1093/infdis/165.3.535

Clements, M. F., Gidwani, K., Kumar, R., Hostomska, J., Dinesh, D. S., Kumar, V., et al. (2010). Measurement of recent exposure to Phlebotomus argentipes, the vector of Indian visceral Leishmaniasis, by using human antibody responses to sand fly saliva. Am. J. Trop. Med. Hyg. 82, 801-807. doi: 10.4269/ajtmh.2010.09-0336

Collin, N., Assumpcao, T. C., Mizurini, D. M., Gilmore, D. C., DutraOliveira, A., Kotsyfakis, M., et al. (2012). Lufaxin, a novel factor Xa inhibitor from the salivary gland of the sand fly Lutzomyia longipalpis blocks protease-activated receptor 2 activation and inhibits inflammation and thrombosis in vivo. Arterioscler. Thromb. Vasc. Biol. 32, 2185-2198. doi: 10.1161/ATVBAHA.112.253906
Collin, N., Gomes, R., Teixeira, C., Cheng, L., Laughinghouse, A., Ward, J. M., et al. (2009). Sand fly salivary proteins induce strong cellular immunity in a natural reservoir of visceral Leishmaniasis with adverse consequences for Leishmania. PLoS Pathog. 5:e1000441. doi: 10.1371/journal.ppat.1000441

Costa, D. L., Rocha, R. L., Carvalho, R. M., Lima-Neto, A. S., Harhay, M. O., Costa, C. H., et al. (2013). Serum cytokines associated with severity and complications of kala-azar. Pathog. Glob. Health 107, 78-87. doi: 10.1179/2047773213Y.0000000078

Courtin, F., Camara, M., Rayaisse, J. B., Kagbadouno, M., Dama, E., Camara, O., et al. (2015). Reducing human-tsetse contact significantly enhances the efficacy of sleeping sickness active screening campaigns: a promising result in the context of elimination. PLoS Negl. Trop. Dis. 9:e0003727. doi: 10.1371/journal.pntd.0003727

Dama, E., Cornelie, S., Bienvenu Somda, M., Camara, M., Kambire, R., Courtin, F., et al. (2013a). Identification of Glossina palpalis gambiensis specific salivary antigens: towards the development of a serologic biomarker of human exposure to tsetse flies in West Africa. Microbes Infect. 15, 416-427. doi: 10.1016/j.micinf.2013.03.001

Dama, E., Cornelie, S., Camara, M., Somda, M. B., Poinsignon, A., Ilboudo, H., et al. (2013b). In silico identification of a candidate synthetic peptide (Tsgf11843) to monitor human exposure to tsetse flies in West Africa. PLoS Negl. Trop. Dis. 7:e2455. doi: 10.1371/journal.pntd.0002455

Desjeux, P. (2004). Leishmaniasis: current situation and new perspectives. Comp. Immunol. Microbiol. Infect. Dis. 27, 305-318. doi: 10.1016/j.cimid.2004.03.004

DE F Michelin, A., Perri, S. H., and De Lima, V. M. (2011). Evaluation of TNF-alpha, IL-4, and IL-10 and parasite density in spleen and liver of L. (L.) chagasi naturally infected dogs. Ann. Trop. Med. Parasitol. 105, 373-383. doi: 10.1179/1364859411Y.0000000027

do Nascimento, P. R., Martins, D. R., Monteiro, G. R., Queiroz, P. V., Freire-Neto, F. P., Queiroz, J. W., et al. (2013). Association of pro-inflammatory cytokines and iron regulatory protein 2 (IRP2) with Leishmania burden in canine visceral Leishmaniasis. PLoS ONE 8:e73873. doi: 10.1371/journal.pone.0073873

Dos Santos, P. L., de Oliveira, F. A., Santos, M. L., Cunha, L. C., Lino, M. T., de Oliveira, M. F., et al. (2016). The severity of visceral Leishmaniasis correlates with elevated levels of serum IL-6, IL-27 and sCD14. PLoS Negl. Trop. Dis. 10:e0004375. doi: 10.1371/journal.pntd.0004375

Doucoure, S., and Drame, P. M. (2015). Salivary biomarkers in the control of mosquito-borne diseases. Insects 6, 961-976 doi: 10.3390/insects6040961

Doucoure, S., Mouchet, F., Cournil, A., Le Goff, G., Cornelie, S., Roca, Y., et al. (2012). Human antibody response to Aedes aegypti saliva in an urban population in Bolivia: a new biomarker of exposure to Dengue vector bites. Am. J. Trop. Med. Hyg. 87, 504-510. doi: 10.4269/ajtmh.2012.11-0477

Duthie, M. S., Guderian, J., Vallur, A., Bhatia, A., Lima dos Santos, P., Vieira de Melo, E., et al. (2014). Alteration of the serum biomarker profiles of visceral Leishmaniasis during treatment. Eur. J. Clin. Microbiol. Infect. Dis. 33, 639-649. doi: 10.1007/s10096-013-1999-1

Esch, K. J., Juelsgaard, R., Martinez, P. A., Jones, D. E., and Petersen, C. A. (2013). Programmed death 1-mediated T cell exhaustion during visceral Leishmaniasis impairs phagocyte function. J. Immunol. 191, 5542-5550. doi: 10.4049/jimmunol.1301810

Foglia Manzillo, V., Di Muccio, T., Cappiello, S., Scalone, A., Paparcone, R., Fiorentino, E., et al. (2013). Prospective study on the incidence and progression of clinical signs in naive dogs naturally infected by Leishmania infantum. PLoS Negl. Trop. Dis. 7:e2225. doi: 10.1371/journal.pntd.0002225

Gautam, S., Kumar, R., Maurya, R., Nylen, S., Ansari, N., Rai, M., et al. (2011). IL-10 neutralization promotes parasite clearance in splenic aspirate cells from patients with visceral Leishmaniasis. J. Infect. Dis. 204, 1134-1137. doi: 10.1093/infdis/jir461

Gidwani, K., Picado, A., Rijal, S., Singh, S. P., Roy, L., Volfova, V., et al. (2011). Serological markers of sand fly exposure to evaluate insecticidal nets against visceral Leishmaniasis in India and Nepal: a clusterrandomized trial. PLoS Negl. Trop. Dis. 5:e1296. doi: 10.1371/journal.pntd.00 01296

Gomes, R. B., Brodskyn, C., de Oliveira, C. I., Costa, J., Miranda, J. C., Caldas, A., et al. (2002). Seroconversion against Lutzomyia longipalpis saliva concurrent with the development of anti-Leishmania chagasi delayed-type hypersensitivity. J. Infect. Dis. 186, 1530-1534. doi: 10.1086/3 44733 
Hostomska, J., Rohousova, I., Volfova, V., Stanneck, D., Mencke, N., and Volf, P. (2008). Kinetics of canine antibody response to saliva of the sand fly Lutzomyia longipalpis. Vector Borne Zoonotic Dis. 8, 443-450. doi: 10.1089/vbz.2007.0214

Ibarra-Meneses, A. V., Sanchez, C., Alvar, J., Moreno, J., and Carrillo, E. (2017). Monocyte chemotactic protein 1 in plasma from soluble Leishmania antigen-stimulated whole blood as a potential biomarker of the cellular immune response to Leishmania infantum. Front. Immunol. 8:1208. doi: 10.3389/fimmu.2017.01208

Kaye, P., and Scott, P. (2011). Leishmaniasis: complexity at the host-pathogen interface. Nat. Rev. Microbiol. 9, 604-615. doi: 10.1038/nrmicro2608

Kostalova, T., Lestinova, T., Maia, C., Sumova, P., Vlkova, M., Willen, L., et al. (2017). The recombinant protein $\mathrm{rSP} 03 \mathrm{~B}$ is a valid antigen for screening dog exposure to Phlebotomus perniciosus across foci of canine Leishmaniasis. Med. Vet. Entomol. 31, 88-93. doi: 10.1111/mve.12192

Kostalova, T., Lestinova, T., Sumova, P., Vlkova, M., Rohousova, I., Berriatua, E., et al. (2015). Canine antibodies against salivary recombinant proteins of Phlebotomus perniciosus: a longitudinal study in an endemic focus of canine Leishmaniasis. PLoS Negl. Trop. Dis. 9:e0003855. doi: 10.1371/journal.pntd.0003855

Lainson, R., and Rangel, E. F. (2005). Lutzomyia longipalpis and the eco-epidemiology of American visceral Leishmaniasis, with particular reference to Brazil: a review. Mem. Inst. Oswaldo Cruz 100, 811-827. doi: 10.1590/S0074-02762005000800001

Lefevre, L., Lugo-Villarino, G., Meunier, E., Valentin, A., Olagnier, D., Authier, H., et al. (2013). The C-type lectin receptors dectin-1, MR, and SIGNR3 contribute both positively and negatively to the macrophage response to Leishmania infantum. Immunity 38, 1038-1049. doi: 10.1016/j.immuni.2013.04.010

Lestinova, T., Rohousova, I., Sima, M., de Oliveira, C. I., and Volf, P. (2017). Insights into the sand fly saliva: blood-feeding and immune interactions between sand flies, hosts, and Leishmania. PLoS Negl. Trop. Dis. 11:e0005600. doi: 10.1371/journal.pntd.0005600

Lima, I. S., Silva, J. S., Almeida, V. A., Junior, F. G., Souza, P. A., Larangeira, D. F., et al. (2014). Severe clinical presentation of visceral Leishmaniasis in naturally infected dogs with disruption of the splenic white pulp. PLoS ONE 9:e87742. doi: 10.1371/journal.pone.0087742

Manna, L., Reale, S., Picillo, E., Vitale, F., and Gravino, A. E. (2008). Interferon-gamma (INF-gamma), IL4 expression levels and Leishmania DNA load as prognostic markers for monitoring response to treatment of Leishmaniotic dogs with miltefosine and allopurinol. Cytokine 44, 288-292. doi: 10.1016/j.cyto.2008.08.017

Manna, L., Reale, S., Vitale, F., and Gravino, A. E. (2009). Evidence for a relationship between Leishmania load and clinical manifestations. Res. Vet. Sci. 87:76-78. doi: 10.1016/j.rvsc.2008.12.009

Marzouki, S., Abdeladhim, M., Abdessalem, C. B., Oliveira, F., Ferjani, B., Gilmore, D., et al. (2012). Salivary antigen SP32 is the immunodominant target of the antibody response to Phlebotomus papatasi bites in humans. PLoS Negl. Trop. Dis. 6:e1911. doi: 10.1371/journal.pntd.0001911

Marzouki, S., Kammoun-Rebai, W., Bettaieb, J., Abdeladhim, M., Hadj Kacem, S., Abdelkader, R., et al. (2015). Validation of recombinant salivary protein PpSP32 as a suitable marker of human exposure to Phlebotomus papatasi, the vector of Leishmania major in Tunisia. PLoS Negl. Trop. Dis. 9:e0003991. doi: 10.1371/journal.pntd.0003991

Mendes-Sousa, A. F., do Vale, V. F., Silva, N. C. S., Guimaraes-Costa, A. B., Pereira, M. H., Sant'Anna, M. R. V., et al. (2017). The Sand fly salivary protein lufaxin inhibits the early steps of the alternative pathway of complement by direct binding to the proconvertase C3b-B. Front. Immunol. 8:1065. doi: 10.3389/fimmu.2017.01065

Mondragon-Shem, K., Al-Salem, W. S., Kelly-Hope, L., Abdeladhim, M., AlZahrani, M. H., Valenzuela, J. G., et al. (2015). Severity of old world cutaneous Leishmaniasis is influenced by previous exposure to sandfly bites in Saudi Arabia. PLoS Negl. Trop. Dis. 9:e0003449. doi: 10.1371/journal.pntd.0003449

Moreira, P. R., Fernando, F. S., Montassier, H. J., Andre, M. R., and de Oliveira Vasconcelos, R. (2016). Polarized M2 macrophages in dogs with visceral Leishmaniasis. Vet. Parasitol. 226, 69-73. doi: 10.1016/j.vetpar.2016.06.032

Mukbel, R. M., Khasharmeh, R. H., Hijjawi, N. S., Khalifeh, M. S., Hatmal, M. M., and McDowell, M. A. (2016). Human immune response to salivary proteins of wild-caught Phlebotomus papatasi. Parasitol. Res. 115, 3345-3355. doi: 10.1007/s00436-016-5094-2
Nascimento, M. S., Carregaro, V., Lima-Junior, D. S., Costa, D. L., Ryffel, B., Duthie, M. S., et al. (2015). Interleukin 17A acts synergistically with interferon gamma to promote protection against Leishmania infantum infection. J. Infect. Dis. 211, 1015-1026. doi: 10.1093/infdis/jiu531

Noukpo, M. H., Damien, G. B., Elanga-N'Dille, E., Sagna, A. B., Drame, P. M., Chaffa, E., et al. (2016). Operational assessment of long-lasting insecticidal nets by using an anopheles salivary biomarker of human-vector contact. Am. J. Trop. Med. Hyg. 95, 1376-1382. doi: 10.4269/ajtmh.15-0541

Nylen, S., and Sacks, D. (2007). Interleukin-10 and the pathogenesis of human visceral Leishmaniasis. Trends Immunol. 28, 378-384. doi: 10.1016/j.it.2007.07.004

Parham, P. E., Waldock, J., Christophides, G. K., and Michael, E. (2015). Climate change and vector-borne diseases of humans. Philos. Trans. R. Soc. Lond. B. Biol. Sci. 370:20140377. doi: 10.1098/rstb.2014.0377

Pitta, M. G., Romano, A., Cabantous, S., Henri, S., Hammad, A., Kouriba, B., et al. (2009). IL-17 and IL-22 are associated with protection against human kala azar caused by Leishmania donovani. J. Clin. Invest. 119, 2379-2387. doi: 10.1172/JCI38813

Poinsignon, A., Remoue, F., Rossignol, M., Cornelie, S., Courtin, D., Grebaut, P., et al. (2008). Human IgG antibody response to Glossina saliva: an epidemiologic marker of exposure to Glossina bites. Am. J. Trop. Med. Hyg. 78, 750-753.

Purse, B. V., Carpenter, S., Venter, G. J., Bellis, G., and Mullens, B. A. (2015). Bionomics of temperate and tropical Culicoides midges: knowledge gaps and consequences for transmission of Culicoides-borne viruses. Annu. Rev. Entomol. 60, 373-392. doi: 10.1146/annurev-ento-010814-020614

Quinnell, R. J., Soremekun, S., Bates, P. A., Rogers, M. E., Garcez, L. M., Courtenay, O., et al. (2018). Antibody response to sand fly saliva is a marker of transmission intensity but not disease progression in dogs naturally infected with Leishmania infantum. Parasit. Vectors 11:7. doi: 10.1186/s13071-017-2587-5

Reis, A. B., Giunchetti, R. C., Carrillo, E., Martins-Filho, O. A., and Moreno, J. (2010). Immunity to Leishmania and the rational search for vaccines against canine Leishmaniasis. Trends Parasitol. 26, 341-349. doi: $10.1016 /$ j.pt.2010.04.005

Ribeiro, J. M., and Francischetti, I. M. (2003). Role of arthropod saliva in blood feeding: sialome and post-sialome perspectives. Annu. Rev. Entomol. 48:73-88. doi: 10.1146/annurev.ento.48.060402.102812

Rodriguez-Cortes, A., Carrillo, E., Martorell, S., Todoli, F., Ojeda, A., Martinez-Florez, A., et al. (2016). Compartmentalized immune response in Leishmaniasis: changing patterns throughout the disease. PLoS ONE 11:e0155224. doi: 10.1371/journal.pone.0155224

Rohousova, I., Ozensoy, S., Ozbel, Y., and Volf, P. (2005). Detection of speciesspecific antibody response of humans and mice bitten by sand flies. Parasitology 130, 493-499. doi: 10.1017/S003118200400681X

Rohousova, I., Talmi-Frank, D., Kostalova, T., Polanska, N., Lestinova, T. Kassahun, A., et al. (2015). Exposure to Leishmania spp. and sand flies in domestic animals in northwestern Ethiopia. Parasit. Vectors 8:360. doi: 10.1186/s13071-015-0976-1

Roque, A. L., and Jansen, A. M. (2014). Wild and synanthropic reservoirs of Leishmania species in the Americas. Int. J. Parasitol. Parasites Wildl. 3, 251-262. doi: 10.1016/j.ijppaw.2014.08.004

Schriefer, A., Barral, A., Carvalho, E. M., and Barral-Netto, M. (1995). Serum soluble markers in the evaluation of treatment in human visceral Leishmaniasis. Clin. Exp. Immunol. 102, 535-540. doi: 10.1111/j.1365-2249.1995.tb03849.x

Sima, M., Ferencova, B., Warburg, A., Rohousova, I., and Volf, P. (2016). Recombinant salivary proteins of Phlebotomus orientalis are suitable antigens to measure exposure of domestic animals to sand fly bites. PLoS Negl. Trop. Dis. 10:e0004553. doi: 10.1371/journal.pntd.0004553

Simo, L., Kazimirova, M., Richardson, J., and Bonnet, S. I. (2017). The essential role of tick salivary glands and saliva in tick feeding and pathogen transmission. Front. Cell. Infect. Microbiol. 7:281. doi: 10.3389/fcimb.2017. 00281

Soares, B. R., Souza, A. P., Prates, D. B., de Oliveira, C. I., Barral-Netto, M., Miranda, J. C., et al. (2013). Seroconversion of sentinel chickens as a biomarker for monitoring exposure to visceral Leishmaniasis. Sci. Rep. 3:2352. doi: 10.1038/srep02352

Solca, M. S., Andrade, B. B., Abbehusen, M. M., Teixeira, C. R., Khouri, R., Valenzuela, J. G., et al. (2016). Circulating biomarkers of immune 
activation, oxidative stress and inflammation characterize severe canine visceral Leishmaniasis. Sci. Rep. 6:32619. doi: 10.1038/srep32619

Souza, A. P., Andrade, B. B., Aquino, D., Entringer, P., Miranda, J. C., Alcantara, R., et al. (2010). Using recombinant proteins from Lutzomyia longipalpis saliva to estimate human vector exposure in visceral Leishmaniasis endemic areas. PLoS Negl. Trop. Dis. 4:e649. doi: 10.1371/journal.pntd.0000649

Teixeira, C., Gomes, R., Collin, N., Reynoso, D., Jochim, R., Oliveira, F., et al. (2010). Discovery of markers of exposure specific to bites of Lutzomyia longipalpis, the vector of Leishmania infantum chagasi in Latin America. PLoS Negl. Trop. Dis. 4:e638. doi: 10.1371/journal.pntd.0000638

Vlkova, M., Rohousova, I., Drahota, J., Stanneck, D., Kruedewagen, E. M., Mencke, N., et al. (2011). Canine antibody response to Phlebotomus perniciosus bites negatively correlates with the risk of Leishmania infantum transmission. PLoS Negl. Trop. Dis. 5:e1344. doi: 10.1371/journal.pntd.0001344

Werneck, G. L., Batista, M. S., Gomes, J. R., Costa, D. L., and Costa, C. H. (2003). Prognostic factors for death from visceral Leishmaniasis in Teresina, Brazil. Infection 31, 174-177. doi:10.1007/s15010-003-3139-9

WHO/Leishmaniasis (2014). Leishmaniasis: magnitude of the problem. Available online at: http://www.who.int/leishmaniasis/burden/magnitude/burden magnitude/en/index.html
Ya-Umphan, P., Cerqueira, D., Parker, D. M., Cottrell, G., Poinsignon, A., Remoue, F., et al. (2017). Use of an anopheles salivary biomarker to assess malaria transmission risk along the thailand-myanmar border. J. Infect. Dis. 215, 396-404. doi: 10.1093/infdis/jiw543

Zhao, X., Alves e Silva, T. L., Cronin, L., Savage, A. F., O'Neill, M., Nerima, B., et al. (2015). Immunogenicity and serological cross-reactivity of saliva proteins among different tsetse species. PLoS Negl. Trop. Dis. 9:e0004038. doi: 10.1371/journal.pntd.00 04038

Conflict of Interest Statement: The authors declare that the research was conducted in the absence of any commercial or financial relationships that could be construed as a potential conflict of interest.

Copyright $\odot 2018$ Brodskyn and Kamhawi. This is an open-access article distributed under the terms of the Creative Commons Attribution License (CC BY). The use, distribution or reproduction in other forums is permitted, provided the original author(s) and the copyright owner(s) are credited and that the original publication in this journal is cited, in accordance with accepted academic practice. No use, distribution or reproduction is permitted which does not comply with these terms. 